\title{
Primary malignant perivascular epithelioid cell neoplasm (PEComa) of the bone mimicking granular cell tumor in core biopsy: A case report and literature review
}

\author{
SAM SADIGH $^{1^{*}}$, PREYA SHAH $^{2 *}$, KRISTY WEBER ${ }^{3}$, RONNIE SEBRO $^{2}$ and PAUL J. ZHANG ${ }^{1}$ \\ Departments of ${ }^{1}$ Pathology, ${ }^{2}$ Radiology and ${ }^{3}$ Orthopaedic Surgery, \\ Hospital of The University of Pennsylvania, Philadelphia, PA 19104, USA
}

Received March 25, 2017; Accepted July 3, 2017

DOI: $10.3892 / \mathrm{ol} .2017 .7662$

\begin{abstract}
The present study investigated the case of a 46-year-old female with primary malignant perivascular epithelioid cell neoplasm (PEComa) of the femur. The patient presented with a 5-month history of right distal thigh pain following trauma. Radiographs of the right distal femur revealed a mixed lytic and sclerotic lesion with subtle areas of cortical destruction and soft tissue extension, consistent with an aggressive tumor. A core biopsy revealed an epithelioid tumor with granular cell features, but a definitive diagnosis could not be made. Due to the aggressive features on radiologic evaluation, the patient underwent a resection of the distal femur and reconstruction with a distal femoral megaprosthesis and hinged knee replacement. The post-resection pathology led to a final diagnosis of primary bone PEComa, with histologic features including epithelioid, granular cell and spindled cell morphologies and biphasic immunoreactivity for melanocytic and smooth muscle markers. The large tumor size $(>5 \mathrm{~cm})$, rapid mitotic rate, infiltrative growth pattern, high nuclear grade and cellularity, and the presence of necrosis rendered this a malignant PEComa. The present study discussed the case, including radiographic (radiographs, magnetic resonance imaging and positron emission tomography scans) and histologic appearance and a literature review.
\end{abstract}

Correspondence to: Dr Ronnie Sebro, Department of Radiology, Hospital of The University of Pennsylvania, 3400 Spruce Street, Philadelphia, PA 19104, USA

E-mail: ronnie.sebro@uphs.upenn.edu

Professor Paul J. Zhang, Department of Pathology, Hospital of The University of Pennsylvania, 3400 Spruce Street, Philadelphia, PA 19104, USA

E-mail: paul.zhang2@uphs.upenn.edu

*Contributed equally

Key words: tumor, radiograph, magnetic resonance imaging, MRI, perivascular epithelioid cell neoplasm, PET, positron emission tomography, PEComa

\section{Introduction}

Perivascular epithelioid cell (PEC) neoplasms (PEComas) are rare mesenchymal tumors composed of PECs. PEC, a cell type that has no known normal counterpart, was coined by Bonetti et al (1) in 1992 in order to describe tumor cells with an epithelioid appearance, perivascular distribution, and co-expression of myogenic and melanocytic markers, most classically Human Melanoma Black 45 (HMB45). Histologically, PEComas typically exhibit both epithelioid and spindled cell morphology, and can be either benign or malignant (2).

While the most prevalent tumors in the PEComa family are angiomyolipoma (AML), lymphangioleiomyomatosis (LAM) and clear-cell (sugar) tumor (CCST) of the lung, other significantly less common manifestations of PEComa have been detected at a variety of visceral and somatic sites throughout the body (3-5). Primary PEComa of the bone is particularly rare, with only 12 reported cases in the literature (6-14). The present study investigated a case of malignant primary bone PEComa presenting in the right distal femur of a 46-year-old female and described the clinical, radiologic and histologic malignant features, in addition to a review of the relevant literature.

\section{Case report}

A 46-year-old female with no significant past medical history presented with a 5-month history of right distal thigh pain following trauma. The patient presented to urgent care in 2016 for evaluation following the trauma and obtained radiographs of the right knee and distal femur (Fig. 1A and B). These radiographs revealed a mixed, lytic and sclerotic lesion involving the distal femoral metadiaphysis extending into the epiphysis. The patient's pain progressed and she returned three months later for a repeat evaluation. At that time, the patient reported $5 / 10$ distal thigh pain that was throbbing and aching. The pain was constant. The pain was worse with activity and relieved with rest and non-steroidal anti-inflammatory drugs.

Radiographs of the right distal femur were repeated (Fig. 2A and B), which revealed the lesion to be grossly similar in size when compared with prior radiographs, though with increased cortical scalloping. The patient was referred for 
further evaluation with magnetic resonance imaging (MRI). The MRI was obtained using a 0.3T Hitachi Airis II scanner (Hitachi Medical Corporation, Tokyo, Japan).

Sagittal T1-weighted sequences were obtained using repetition time (TR) of $900 \mathrm{~ms}$, echo time (TE) of $12.6 \mathrm{~ms}$, slice thickness of $4 \mathrm{~mm}$, interslice gap of $1 \mathrm{~mm}$ and acquisition matrix of 256x158 (Fig. 3A). T2-weighted sequences were obtained with TR/TE 6182/125 ms, slice thickness of $4 \mathrm{~mm}$, interslice gap of $1 \mathrm{~mm}$ and acquisition matrix of 256x144 (Fig. 3B). The lesion was heterogeneous in T2 signal with three distinct signal intensities noted. The MRI demonstrated areas of cortical destruction with soft tissue extension consistent with an aggressive lesion (Fig. 3B). Coronal T1-weighted sequences (TR/TE 490/15 ms, slice thickness $4 \mathrm{~mm}$, interslice gap of $0.5 \mathrm{~mm}$ and acquisition matrix 256x160) exhibited a heterogeneously $\mathrm{T} 1$ hypointense and isointense lesion (Fig. 3C). The patient was referred for computed tomography (CT)-guided core-needle biopsy of the lesion at the Hospital of the University of Pennsylvania (Philadelphia, PA, USA). Histologic evaluation of the area of the lesion that had been sampled with the core needle biopsy (Fig. 4A-C) revealed a cytologically bland proliferation of cells, with ample bright granular eosinophilic cytoplasm and dark even chromatin with few scattered enlarged nuclei with inclusions. The cells were in a nested configuration with a rich vascular stroma, and other areas had extensive stromal hyalinization. There was no necrosis or mitotic activity. The morphologic impression was that of a granular cell tumor, although an S100 immunostain was negative and a subsequent extensive immunohistochemical panel was unrevealing. Considering the overall features, the core needle biopsy diagnosis was favored to be an unusual (S100 negative) granular cell neoplasm with extensive stromal hyalinization. Since malignant granular cell tumors are ${ }^{18}$ fluorodeoxyglucose (FDG)-avid, a PET/CT was performed to evaluate FDG-avidity and assess for additional lesions (Fig. 5). The PET/CT indicated an FDG-avid lesion with a maximum SUV of 28.6, so the lesion was treated as a malignant granular cell neoplasm.

The distal femur lesion was resected with negative margins and the resection gap reconstructed with a distal femoral megaprosthesis and hinged knee replacement (Fig. 6A and B). Gross examination of the tumor revealed a $6.9 \times 3.9 \times 2.9 \mathrm{~cm}$ irregular, heterogeneous, firm lesion with an appearance varying from beige to gray in color (Fig. 7A and B). The lesion occupied the majority of the intramedullary cavity with an area of posterior endosteal scalloping and soft tissue extension through the cortex, as noted on prior MRI.

Histology from the resected specimen revealed a heterogenous morphology with various patterns. A number of areas had plump, epithelioid cells with granular cytoplasm, round medium-large nuclei and distinct cell borders. This was concordant with the previous biopsy (Fig. 8A). These cells were predominantly arranged in a nested architecture with individual nests separated from each other by a vascular or hyalinizing stroma, and in areas with a vaguely storiform pattern (Fig. 8B and C). At least focally, characteristic thin vascular spaces were observed, around which tumor cells were arranged tightly or in a radiating manner (Fig. 8D). Other regions had an infiltrative pattern with a spindled morphology, with medium sized spindled cells arranged in interweaving fascicles and containing fibrillar or granular eosinophilic cytoplasm with elongated, ovoid nuclei with prominent nucleoli (Fig. 8E). There were cells present that exhibited marked nuclear pleomorphism (Fig. 8F). Mitotic activity was highest in very cellular epithelioid and spindled areas with $>5$ mitoses per high power field (Fig. 8G and H). Focal coagulative tumor necrosis was also present.

Tumor cells revealed positivity for smooth muscle actin, Caldesmin, melanoma antigen, epithelial membrane antigen and thyroid transcription factor-1 (TTF-1; nuclear), while being negative for pan-cytokeratin, cytokeratin AE1/AE3, cytokeratin Cam5.2, S100, CKIT, discovered on gastrointenstinal stromal tumors protein 1, DOG-1, Desmin, Paired box 8, Signal transducer and activator of transcription 6 , Inhibin, Thyroglobulin, Cyclin D1, microphthalmia associated transcription factor 2 and Human Melanoma Black (HMB45). There was abnormal transcription factor E3 (TFE3; nuclear) expression, although fluorescent in situ hybridization (FISH) did not demonstrate a TFE3 translocation. Due to the negative cytokeratin expression, the TTF-1 reactivity was considered as aberrant reactivity rather than evidence of an epithelial tumor. There was no abnormal loss of SDHB. CD34 and CD31 indicated an extensive vascular network. Based on the morphologic and immunohistochemical findings (co-expression of smooth muscle markers and melanocytic markers), the final pathologic diagnosis on the resected specimen was of a malignant PEComa.

All procedures performed involving human participants were in accordance with the ethical standards of the University of Pennsylvania Health System (Philadelphia, PA, USA) and with the Declaration of Helsinki declaration (1964) and its later amendments or comparable ethical standards. The requirement for informed consent was waived by the local institutional review board.

\section{Discussion}

We present a novel case of primary bone PEComa, localized to the right distal femur. The concept of PEComa is relatively recent, arising within the last 25 years: Following Bonetti's description of PECs in 1992 (1), Zamboni et al (15) coined the term 'PEComa' in 1996 and the World Health Organization first recognized PEComa in 2002 (16). As a result, numerous rare neoplastic disorders, including renal AML (prevalence $\sim 1-2 / 1000$ individuals) (17), pulmonary LAM (prevalence $\sim 1-2 / 1,000,000$ individuals) $(2,18)$ and CCST of the lung (unknown prevalence, $\sim 50$ reported cases) (19-21), now fall within the umbrella term of PEComa. Other forms of PEComa, known as PEC tumor not otherwise specified (PEComa-NOS), are even less common with $<300$ reported cases (22).

Notably, only 12 cases of primary PEComa of the bone have been reported to date, not including the current case (6-14), with 7 of these classified as malignant. They include a wide age range of patients (mean age 49, range 26-93), with tumors most commonly localized to lower extremities (tibia, fibula, femur and acetabulum) but also occurring in the vertebrae and ribs. Table I summarizes the salient details of each case, including presentation, diagnostic features, treatment and outcomes. Table I also includes details of the present case for comparison. 

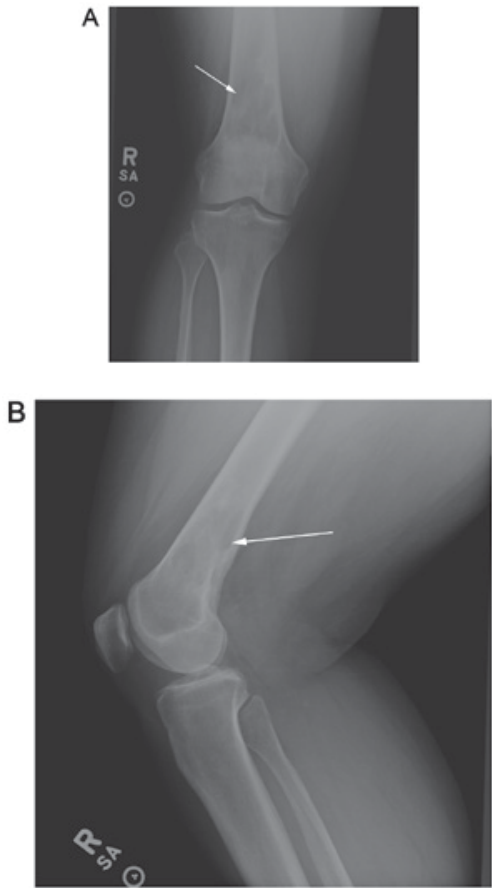

Figure 1. (A) Anterior-posterior and (B) lateral radiographs of the right knee demonstrating a mixed lytic and sclerotic lesion (white arrow) involving the distal femoral metadiaphysis.
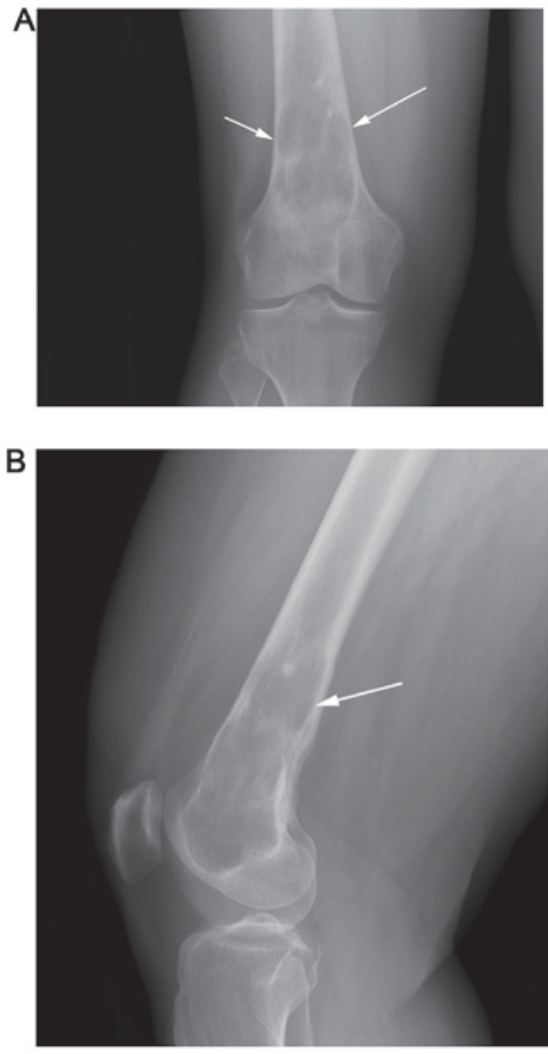

Figure 2. (A) Anterior-posterior and (B) lateral radiographs of the right knee demonstrating a mixed lytic and sclerotic lesion involving the distal femoral metadiaphysis with mild cortical thinning/scalloping (white arrows).

The etiology of PEComa is unclear. There is no normal cell type analogous to the PEC and no identified precursor lesion. Genetically, AML, LAM and CCST are strongly associated
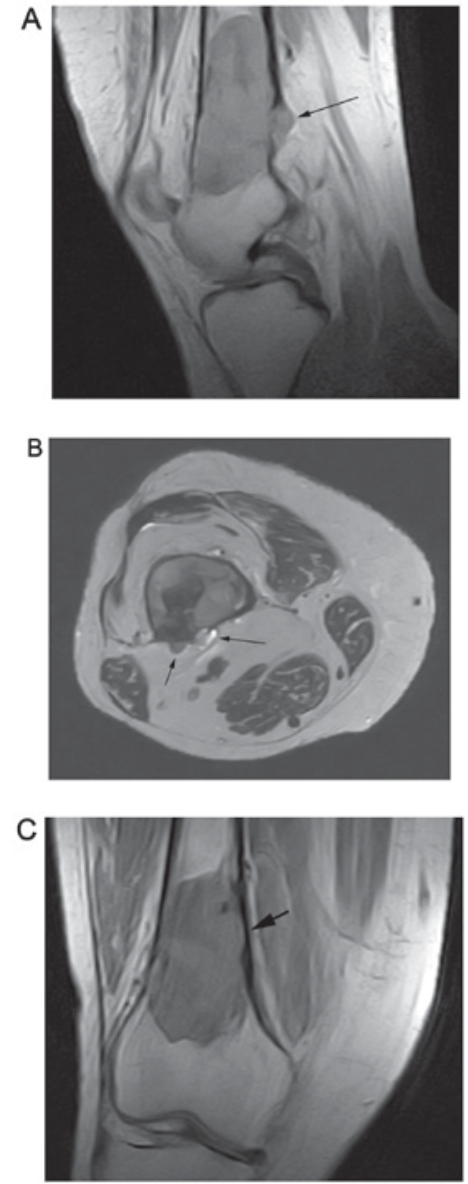

Figure 3. (A) Sagittal proton-density weighted MRI of the right knee demonstrating a heterogeneous lesion involving the distal femoral metadiaphysis with cortical destruction and an extra-osseous soft tissue component (black arrow). (B) Axial T2-weighted MRI of the right knee indicating a T2-heterogeneous lesion with extraosseous extension through the posterior femoral cortex (black arrows). (C) Coronal T1-weighted MRI of the right knee illustrating a heterogeneously $\mathrm{T} 1$ isointense lesion with scalloping of the medial femoral cortex (black arrow). MRI, magnetic resonance image.

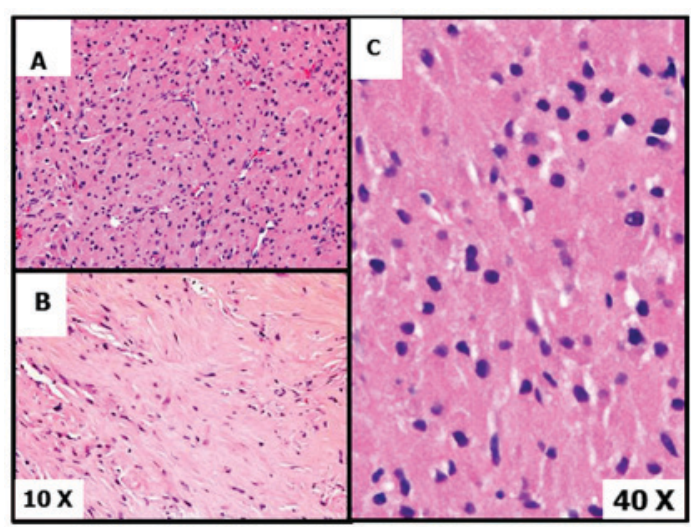

Figure 4. (A) Low power hematoxylin and eosin stains of the core biopsy revealed a proliferation of cells in a nested configuration with rich vascular stroma, (B) and areas of extensive stromal hyalinization. (C) A high power view revealed cells with ample bright granular eosinophilic cytoplasm and dark even chromatin, with no significant atypia or mitotic activity.

with tuberous sclerosis complex (TSC), an autosomal dominant neurocutaneous disorder caused by TSC1/TSC2 gene mutations and characterized by seizures, intellectual disability and cellular 


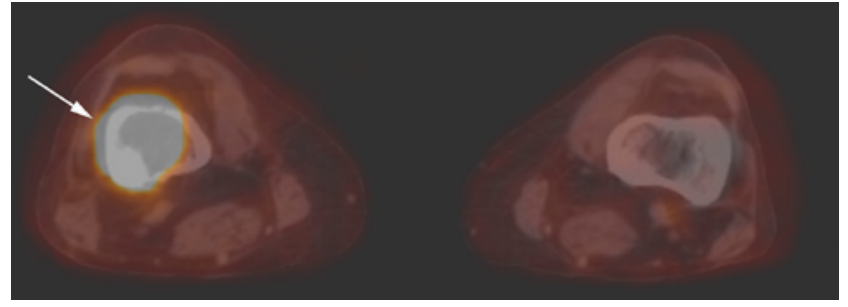

Figure 5. Axial fused positron emission tomography/computed tomography image of the distal femurs indicating the FDG-avid lesion in the distal femur (white arrow).
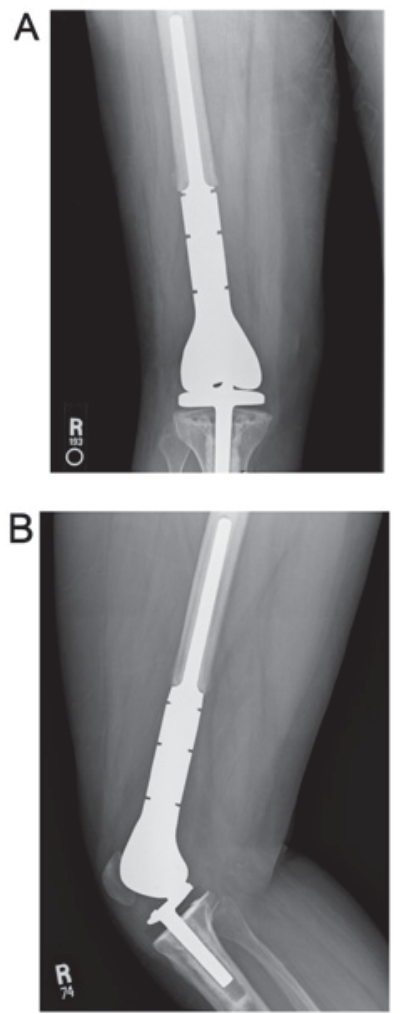

Figure 6. Postoperative (A) anterior-posterior and (B) lateral radiographs of the right knee demonstrating distal femoral megaprosthesis and knee replacement.

proliferations (23-25). However, no similar genetic association has been established in primary bone PEComa. AML, LAM, CCST and numerous forms of PEComa-NOS have a well-documented marked female predominance $(15,17,24,26,27)$, which suggests a potential role of hormones in pathogenesis. However, this trend is not evident in primary bone PEComa (6/13 existing cases, including the present case, are female), though the total number of cases is too few to be conclusive.

In general, PEComas may be asymptomatic or present with nonspecific pain. All existing cases of primary bone PEComa presented with pain and/or swelling, with PEComas of the vertebral column also causing leg weakness due to cord compression $(10,12)$. Diagnostic workup of bone PEComa involves a combination of imaging, histology, and immunohistochemistry. Based on the existing case studies, there appears to be no consistent protocol in terms of imaging workup, though the majority of studies include at least a radiograph,

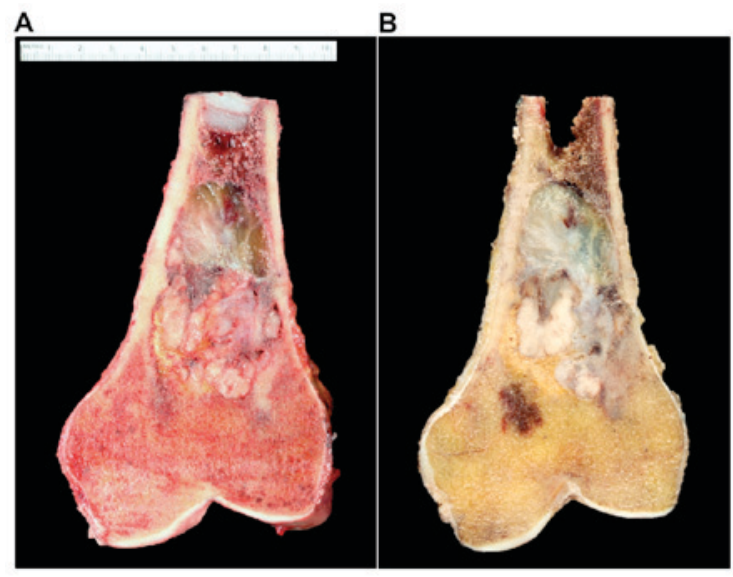

Figure 7. Coronal section of resected distal femoral specimen in the (A) fresh state and (B) post-formalin fixation, depicts a $6.9 \times 3.9 \times 2.9 \mathrm{~cm}$ irregular heterogeneous firm lesion, with appearance varying from white-tan to beige, gray and tan-brown, which occupies the majority of the intramedullary space. There is bone wax at the proximal end of the specimen.

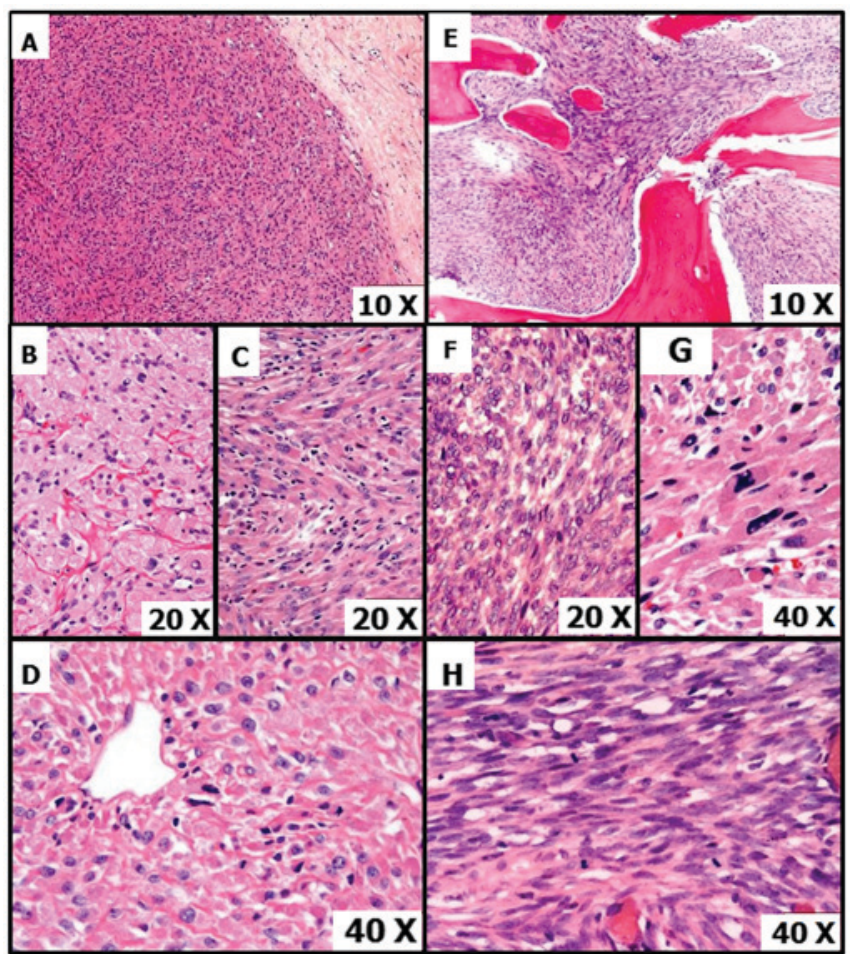

Figure 8. Hematoxylin and eosin stains of the resected specimen demonstrated notable heterogeneous morphologies. (A) Regions corresponding to the biopsied site contain plump, epithelioid cells with granular cytoplasm, round medium to large nuclei, and distinct cell borders that are (B) typically arranged in a nested architecture with a vascular or hyalinizing stroma and (C) in areas with a vaguely storiform pattern. (D) Epithelioid cells arranged about a thin vascular space. (E) Areas with an infiltrative pattern, as well as (F) bizarre atypia and (G) markedly cellular regions were present. (H) A cellular spindled morphology predominated in the infiltrating areas, with medium-sized spindled cells arranged in interweaving fascicles and containing fibrillar or granular eosinophilic cytoplasm with elongated, ovoid nuclei with prominent nucleoli. High mitotic activity is also present.

often followed by CT and/or MRI $(11,12)$. The most common radiographic finding is a mixed lytic and sclerotic lesion, with more aggressive tumors also presenting with soft tissue expansion and cortical destruction on CT or MRI. MRI is likely to 


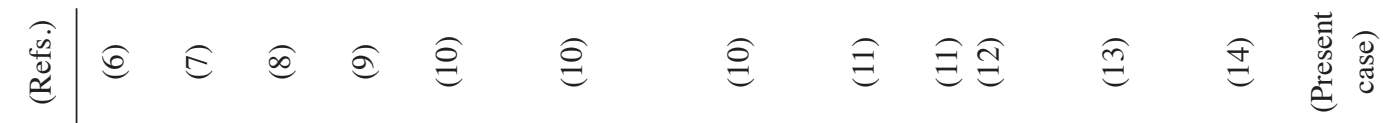

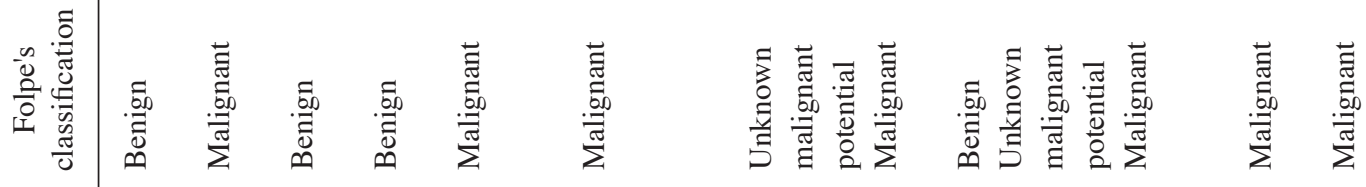

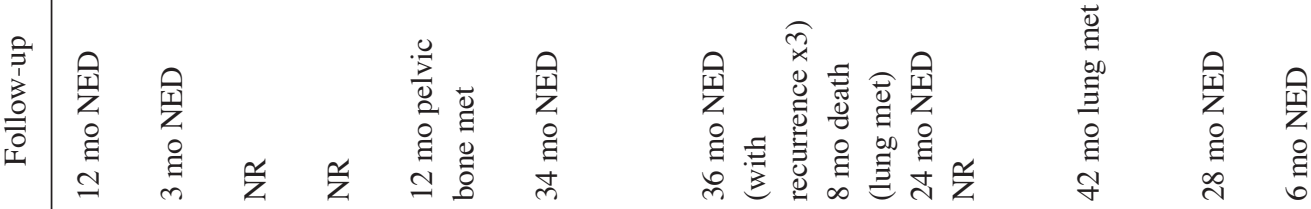

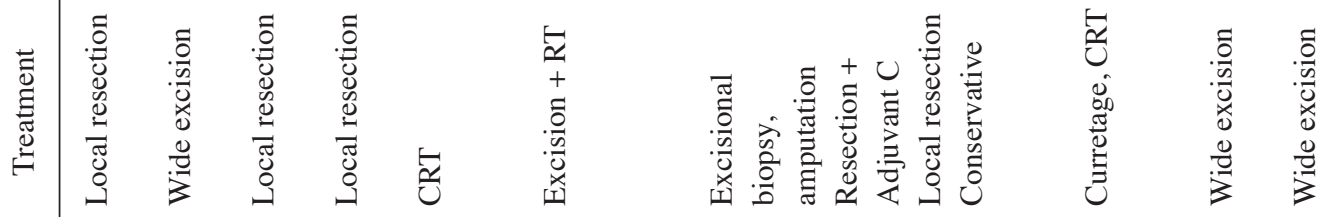

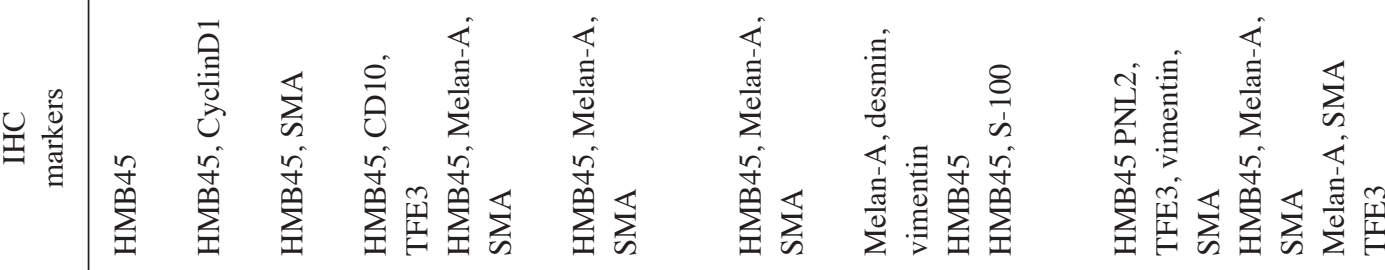

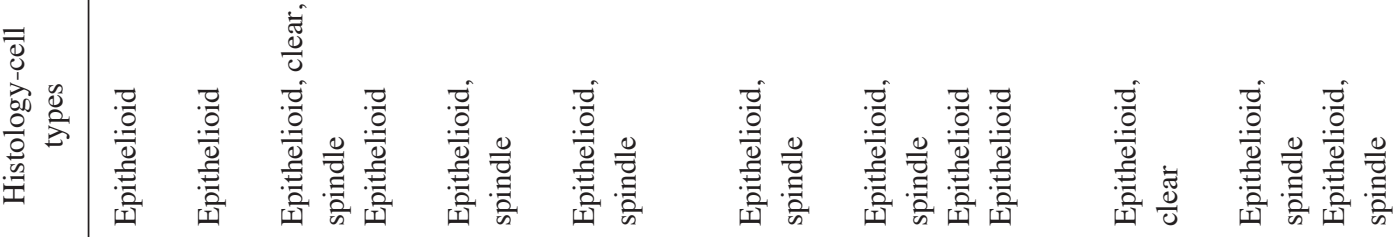

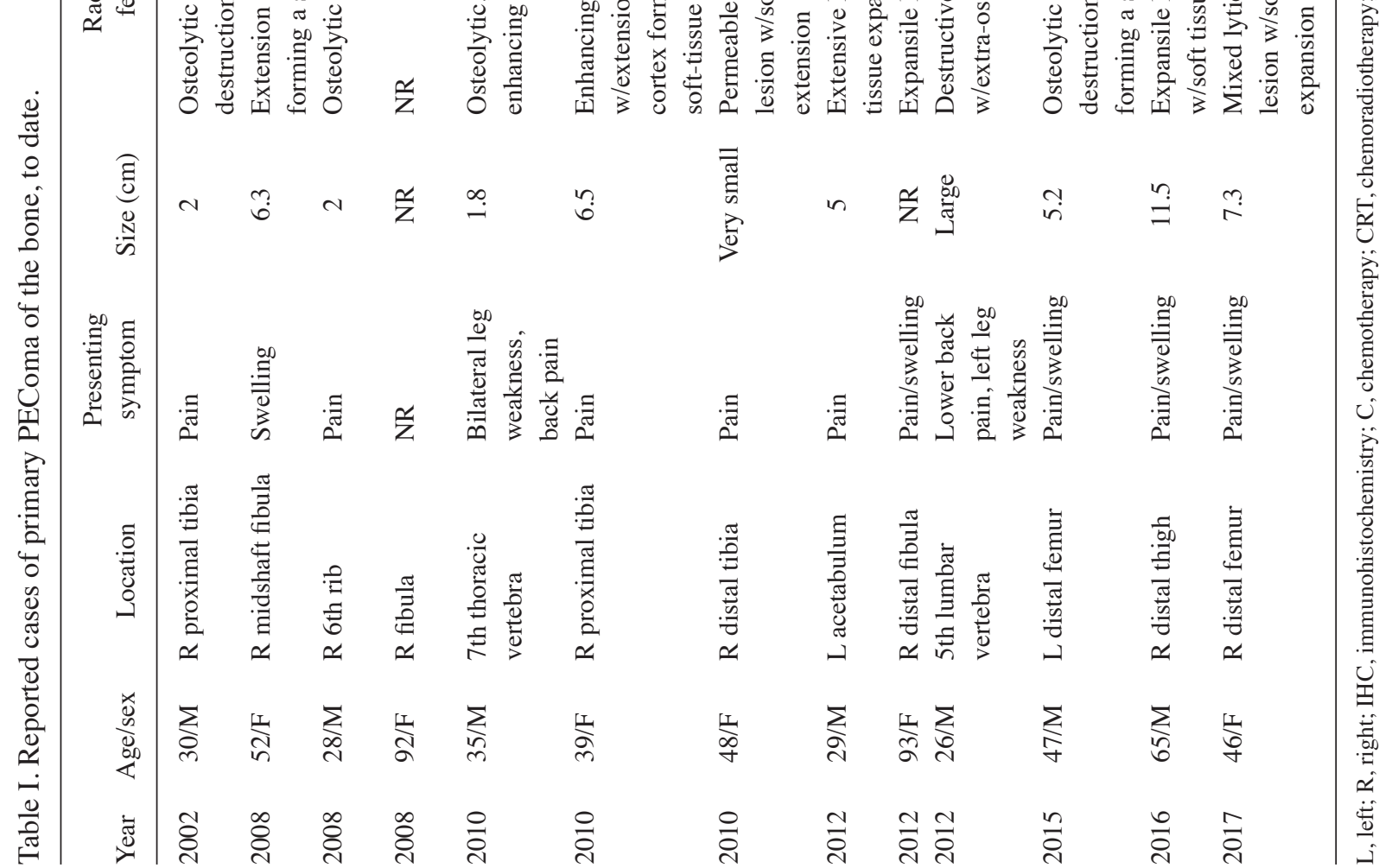


exhibit heterogeneous T1 signal hypointensity on T1-weighted imaging and heterogeneous T2 signal hyperintensity on T2-weighted images $(10,11,13)$. Following analysis of local radiologic findings, patients often undergo systemic clinical and radiologic testing for staging and exclusion of metastatic disease.

In addition to imaging, histological and immunohistochemical studies are crucial for the diagnosis of PEComa. These can be carried out following biopsy, intralesional curettage or wide resection of the tumor. Cytologic findings are significant for epithelioid cells with clear and eosinophilic cytoplasm, intermixed with spindle cells. The cells lie adjacent to thin-walled blood vessels. Other findings common in more aggressive cases are high mitotic activity, nuclear pleomorphism, multinucleated giant cells, stromal hyalinization and focal necrosis $(7,8,10,11,13,14)$. PEComas typically exhibit reactivity to both melanocytic (e.g., HMB45, melan-A) and myogenic (e.g., smooth muscle actin, desmin) markers, but not to epithelial markers. The present case is one of only two reported primary bone PEComas with negative staining for HMB45 but positive staining for melan-A, another melanocytic marker. Also, the current case also positively expressed at least one smooth muscle marker, similar to $7 / 12$ of the prior cases. Notably, this case also stained positively for TFE3, a member of the MiT family of transcription factors, albeit FISH did not demonstrate a translocation. TFE3 positivity has been observed in two prior primary bone PEComa cases and other forms of PEComa. In fact, in a study of TFE3 positivity in PEComa cases, Righi et al (9) proposed that PEComa be added to the MiT family of human tumors, which also includes melanoma, alveolar soft part sarcoma, translocation-associated renal cell carcinomas and clear cell sarcoma of soft tissue (9).

The histologic differential diagnosis for bone PEComa includes metastatic melanoma, metastatic carcinoma (e.g., of renal origin), and leiomyosarcoma. These lesions can have similar cytologic presentations, such as mixed epithelioid and spindle cell morphology, though they lack a perivascular distribution. Certain forms of renal cell carcinoma also exhibit TFE3 nuclear immunoreactivity (9). Alveolar soft part sarcoma (ASPS), which is also commonly reactive to TFE3 and presents with eosinophilic cytoplasm and hypervascularity (28), should also be considered in the differential. Immunohistochemistry is crucial for distinguishing primary bone PEComa from other metastatic disease. In contrast to PEComa, leiomyosarcoma and ASPS lack expression of melanocytic markers, metastatic melanoma lacks expression of smooth muscle markers and expresses S-100, and carcinoma expresses epithelial markers $(2,10,16,26,28)$. Therefore, the trifecta of imaging, histology and immunohistochemistry is essential in PEComa evaluation: While radiologic studies are often the first step of the workup and microscopic examination can provide further insights, immunohistochemistry is required for a definitive diagnosis.

In 2005, Folpe and Kwiatkowski (2) established histologic criteria to classify PEComas as 'benign', 'uncertain malignant potential' or 'malignant'. Specifically, they suggest that a PEComa be classified as malignant if it exhibits at least two of the following features: i) Tumor size $>5 \mathrm{~cm}$; ii) infiltrative growth pattern; iii) high nuclear grade; iv) high cellularity; v) necrosis; vi) mitotic activity $>1 / 50 \mathrm{HPF}$ with subsequent aggressive clinical behavior. Neoplasms fulfilling only one of the six criteria may be characterized as having uncertain malignant potential, while those with none of the features may be considered benign. Based on this system, the current patient's PEComa would be classified as malignant, given its large size, high mitotic rate, infiltrative growth pattern, high nuclear grade and cellularity, and the presence of necrosis. Table I includes malignancy status based on Folpe's criteria for all prior primary bone PEComa cases. It is important to note that malignancy status based on Folpe's histological criteria may not reflect malignancy based on clinical aggressiveness, though the prior cases that involved metastatic disease were all also histologically classified as malignant.

In terms of treatment, resection of bone PEComa is most common and often curative, though chemo- and/or radiotherapy may be necessary in the case of metastatic disease. Prognosis is variable and benign lesions are cured with local resection and malignant lesions develop metastasis in $42.9 \%$ (3/7) of cases from Table I. As evident from Table I, long-term follow-up information is not consistently reported, which can make conclusions regarding treatment outcomes challenging. Additional and longer-term studies of patients with primary PEComa of the bone can further understanding of this exceptionally rare but fascinating disorder.

\section{References}

1. Bonetti F, Pea M, Martignoni G and Zamboni G: PEC and sugar. Am J Surg Pathol 16: 307-308, 1992.

2. Folpe AL and Kwiatkowski DJ: Perivascular epithelioid cell neoplasms: Pathology and pathogenesis. Hum Pathol 41: 1-15, 2010.

3. Martignoni G, Pea M, Reghellin D, Zamboni G and Bonetti F: PEComas: The past, the present and the future. Virchows Arch 452: 119-132, 2008.

4. Pan CC, Yang AH and Chiang H: Malignant perivascular epithelioid cell tumor involving the prostate. Arch Pathol Lab Med 127: E96-E98, 2003.

5. Vang R and Kempson RL: Perivascular epithelioid cell tumor ('PEComa') of the uterus: A subset of HMB-45-positive epithelioid mesenchymal neoplasms with an uncertain relationship to pure smooth muscle tumors. Am J Surg Pathol 26: 1-13, 2002.

6. Insabato L, De Rosa G, Terracciano LM, Fazioli F, Di Santo F and Rosai J: Primary monotypic epithelioid angiomyolipoma of bone. Histopathol 40: 286-290, 2002.

7. Lian DW, Chuah KL, Cheng MH and Yap WM: Malignant perivascular epithelioid cell tumour of the fibula: A report and a short review of bone perivascular epithelioid cell tumour. J Clin Pathol 61: 1127-1129, 2008.

8. Torii I, Kondo N, Takuwa T, Matsumoto S, Okumura Y, Sato A, Tanaka F, Nishigami T, Hasegawa $S$ and Tsujimura T: Perivascular epithelioid cell tumor of the rib. Virchows Arch 452: 697-702, 2008.

9. Righi A, Dimosthenous K and Rosai J: PEComa: Another member of the MiT tumor family? Int J Surg Pathol 16: 16-20, 2008.

10. Yamashita K and Fletcher CD: PEComa presenting in bone: Clinicopathologic analysis of 6 cases and literature review. Am J Surg Pathol 34: 1622-1629, 2010.

11. Desy NM, Bernstein M, Nahal A, Aziz M, Kenan S, Turcotte RE and Kahn LB: Primary perivascular epithelioid cell neoplasm (PEComa) of bone: Report of two cases and review of the literature. Skeletal Radiol 41: 1469-1474, 2012.

12. Kazzaz D, Khalifa M, Alorjan M, Shaw M, Rezajooi K and Saifuddin A: Malignant PEComa of the lumbar vertebra: A rare bone tumour. Skeletal Radiol 41: 1465-1468, 2012.

13. Lao IW, Yu L and Wang J: Malignant perivascular epithelioid cell tumor (PEComa) of the femur: A case report and literature review. Diagn Pathol 10: 54, 2015.

14. Yu H, Zhu X, Sheng H, Gao H, Xiao W and Wang C: Case report primary perivascular epithelioid cell neoplasm of thigh bone: A case report and literature review. Int J Clin Exp Pathol 9: 2487-2491, 2016. 
15. Zamboni G, Pea M, Martignoni G, Zancanaro C, Faccioli G, Gilioli E, Pederzoli P and Bonetti F: Clear cell 'sugar' tumor of the pancreas. A novel member of the family of lesions characterized by the presence of perivascular epithelioid cells. Am J Surg Pathol 20: 722-730, 1996.

16. Fletcher CDM, Unni KK and Mertens F: World Health Organization classification of tumours: Pathology and genetics of tumours of soft tissue and bone. Cancer 177: 1365-1376, 2002.

17. Eble JN: Angiomyolipoma of kidney. Semin Diagn Pathol 15: 21-40, 1998.

18. Urban T, Lazor R, Lacronique J, Murris M, Labrune S, Valeyre D and Cordier JF: Pulmonary lymphangioleiomyomatosis. A study of 69 patients. Groupe d'Etudes et de Recherche sur les Maladies 'Orphelines' Pulmonaires. Medicine (Baltimore) 78: 321-337, 1999.

19. Gaffey MJ, Mills SE, Askin FB, Ross GW, Sale GE, Kulander BG, Visscher DW, Yousem SA and Colby TV: Clear cell tumor of the lung. A clinicopathologic, immunohistochemical, and ultrastructural study of eight cases. Am J Surg Pathol 14: 248-259, 1990

20. Gaffey MJ, Mills SE, Zarbo RJ, Weiss LM and Gown AM Clear cell tumor of the lung. Immunohistochemical and ultrastructural evidence of melanogenesis. Am J Surg Pathol 15: 644-653, 1991

21. Bonetti F, Pea M, Martignoni G, Doglioni C, Zamboni G, Capelli P, Rimondi P and Andrion A: Clear cell ('sugar') tumor of the lung is a lesion strictly related to angiomyolipoma-the concept of a family of lesions characterized by the presence of the perivascular epithelioid cells (PEC). Pathology 26: 230-236, 1994.

22. Bleeker JS, Quevedo JF and Folpe AL: 'Malignant' perivascular epithelioid cell neoplasm: Risk stratification and treatment strategies. Sarcoma 2012: 541626, 2012.
23. Rakowski SK, Winterkorn EB, Paul E, Steele DJ, Halpern EF and Thiele EA: Renal manifestations of tuberous sclerosis complex: Incidence, prognosis, and predictive factors. Kidney Int 70: 1777-1782, 2006

24. Costello LC, Hartman TE and Ryu JH: High frequency of pulmonary lymphangioleiomyomatosis in women with tuberous sclerosis complex. Mayo Clin Proc 75: 591-594, 2000

25. Flieder DB and Travis WD: Clear cell 'sugar' tumor of the lung: Association with lymphangioleiomyomatosis and multifocal micronodular pneumocyte hyperplasia in a patient with tuberous sclerosis. Am J Surg Pathol 21: 1242-1247, 1997.

26. Folpe AL, Mentzel T, Lehr HA, Fisher C, Balzer BL and Weiss SW: Perivascular epithelioid cell neoplasms of soft tissue and gynecologic origin: A clinicopathologic study of 26 cases and review of the literature. Am J Surg Pathol 29: 1558-1575, 2005.

27. Armah HB and Parwani AV: Perivascular epithelioid cell tumor. Arch Pathol Lab Med 133: 648-654, 2009.

28. Folpe AL and Deyrup AT: Alveolar soft-part sarcoma: A review and update. J Clin Pathol 59: 1127-1132, 2006.

This work is licensed under a Creative Commons Attribution-NonCommercial-NoDerivatives 4.0 International (CC BY-NC-ND 4.0) License. 